\title{
Selective adsorption from polymer mixtures on solid surfaces
}

\author{
E.V.Vakarin, M.F.Holovko \\ Institute for Condensed Matter Physics \\ of the National Academy of Sciences of Ukraine, \\ 1 Svientsitskii Str., 79011 Lviv, Ukraine
}

Received October 17, 2001

\begin{abstract}
Selective adsorption from binary polymer mixtures on a crystalline surface is studied in terms of the lattice gas model in conjunction with the results of the association theory. An interplay of the selectivity and the surface induced segregation is shown to result in a variety of competing factors which dominate the adsorption process. The role of the bead diameter, chain length and the surface activity in the preferential adsorption of one component is analyzed. A variation of the selectivity coefficient with the density and composition is also discussed.
\end{abstract}

Key words: adsorption, selectivity, polymer mixtures

PACS: $68.45 . D a$

\section{Introduction}

It is a real pleasure for us to contribute this article to the special issue dedicated to J.P.Badiali, the collaboration with whom has stimulated our activity in the field of adsorption at solid-liquid interfaces.

Adsorption of polymers at solid-liquid interfaces has many important scientific and technological applications [1]. These include colloidal stabilization, flocculation, adhesion, coating or lubrication (see [2] for a recent review). Many polymerization reactions generate samples with a rather broad chain length distribution. Such polydisperse mixtures have additional degrees of freedom connected with an arrangement of different species near the adsorbing surfaces. Also the surface activity in adsorbing different components can be used as a tool for the accumulation of appropriate species.

Adsorption of mixtures was studied within various techniques. A role of the chain length difference was analyzed [3] using a combination of the field theory and the scaling concept. One-dimensional square well mixtures were discussed [4] applied to the adsorption in porous materials. Yethiraj and Hall [5] reported the Monte Carlo computer simulation for hard chain - hard sphere mixtures confined to 
slitlike pores. Honnell and Hall [6] studied the equation of state and local structure of chain molecule mixtures near an impenetrable wall. Based on the generalized Flory ideas, they discussed three approximate equations of state and reported MC computer simulations for density profiles. The main conclusion of both studies was: shorter chains exhibit an enhancement near the wall, but longer polymers tend to be depleted. This is due to the difference in the packing properties of each component near the surface. The segregation can be measured by a difference in partial local densities near the wall. For blends of branched and linear polymers this effect was studied by Yethiraj [7]. He claimed that in a purely entropic case (i.e. without specific chain-chain or chain-wall attraction) the species which form more compact clusters (linear chains) segregate to the surface. The surface induced segregation of linear chains with different lengths and bead diameters was discussed in [8] using the methods of association theory.

Note, however, that most authors studied the adsorption behaviour, induced by confinement (a hard wall or a pore), while the specific adsorption effects were not discussed. In this paper we investigate the adsorption of polymeric mixtures on crystalline lattices. An emphasis is made on a possibility of preferential accumulation of one species - the so-called selectivity. Our main purpose is to analyze an interplay of the selectivity and the surface induced segregation. We discussed the role of the bead diameter, chain length and the surface activity in the preferential adsorption of one component. A variation of the selectivity coefficient with the density and composition is also of interest.

\section{Model}

We consider a two-component hard sphere chain mixture with the bead diameters $d_{A}, d_{B}$ and chain lengths $m_{A}, m_{B}$. The bulk segment number densities of the species are $\varrho_{A}$ and $\varrho_{B}$. Then $\varrho_{A} / m_{A}$ and $\varrho_{B} / m_{B}$ are the number densities of the chains. Therefore, the system is characterized by the following set of parameters: ratio of the bead diameters $d=d_{B} / d_{A}$, chain lengths $m_{A}, m_{B}$, molar fractions $c_{A}=\varrho_{A} /\left(\varrho_{A}+\right.$ $\left.\varrho_{B}\right), c_{B}=1-c_{A}$, and the overall packing fraction $\eta=\pi / 6\left(\varrho_{A} d_{A}^{3}+\varrho_{B} d_{B}^{3}\right)$.

The mixture is near a hard wall (located at $z=0$ ) on which there is a square lattice of sticky sites, modelling the crystalline structure. The adsorption potential $U_{\alpha}\left(\mathbf{r}_{i}\right)$ is defined by

$$
\exp \left(-\beta U_{\alpha}\left(\mathbf{r}_{i}\right)\right)=1+\lambda_{\alpha} \sum_{\mathbf{R}_{m}} \delta\left(\mathbf{R}_{i}-\mathbf{R}_{m}\right) \delta\left(z-d_{\alpha} / 2\right)
$$

where $\mathbf{R}_{i}$ is a projection of $\mathbf{r}_{i}$ onto the wall, $\mathbf{R}_{m}$ is a position of a lattice site and $\lambda_{\alpha}$ are the stickiness parameters which determine the surface activity with respect to different species. In what follows the Greek indices correspond to the species $\alpha, \beta=A, B$ and $i, j, m=1 \ldots N$ ( $N$ is the number of adsorbing sites). 


\section{Theory}

The singular one-body potential allows one to perform an exact integration in the partition function that can be expressed in terms of the $n$-body distribution functions $\rho_{\alpha \beta}\left(\mathbf{R}_{1}, \ldots, \mathbf{R}_{n}\right)$ for the reference state, i.e., the state without the adsorbing potential. In such a way we have an infinite series on $\lambda_{\alpha}$ including the correlations of all orders for the reference state. If only pair interactions are taken into account, then the problem can be mapped onto the lattice gas model [9] with pairwise interactions. The partition function is given by

$$
\Xi=\sum_{t_{i}^{\alpha}} \exp \left(-H_{\mathrm{LG}} / k T\right)
$$

with $t_{i}^{\alpha}=0$ or 1 being the set of occupation numbers. The Hamiltonian is written as

$$
H_{\mathrm{LG}}=\sum_{i j} \sum_{\alpha \beta} W_{\alpha \beta}\left(\mathbf{R}_{i}, \mathbf{R}_{j}\right) t_{i}^{\alpha} t_{j}^{\beta}-\sum_{i, \alpha} \mu_{\alpha}\left(\mathbf{R}_{i}\right) t_{i}^{\alpha},
$$

where the chemical potential $\mu_{\alpha}\left(\mathbf{R}_{i}\right)$ and the pair interaction $W_{\alpha \beta}\left(\mathbf{R}_{i} \mathbf{R}_{j}\right)$ are closely connected with the properties of the fluid in the bulk phase. Namely,

$$
\begin{aligned}
\mu_{\alpha}\left(\mathbf{R}_{i}\right) & =k T \ln \left(\lambda_{\alpha} \rho_{\alpha}\left(\mathbf{R}_{i}\right) d_{\alpha}^{3}\right), \\
W_{\alpha \beta}\left(\mathbf{R}_{i}, \mathbf{R}_{j}\right) & =-k T \ln \left(G_{\alpha \beta}\left(\mathbf{R}_{i}, \mathbf{R}_{j}\right)\right),
\end{aligned}
$$

where $\rho_{\alpha}\left(\mathbf{R}_{i}\right)$ and $G_{\alpha \beta}\left(\mathbf{R}_{i}, \mathbf{R}_{j}\right)$ are respectively the one-body and pair correlation functions for the reference state. This representation implies that the competitive adsorption and other two-dimensional processes should depend on the properties of the third dimension. Such an approach was successfully applied to the adsorption of the network forming fluids [10], predicting a significant role of the cooperative effects. Choosing properly the reference state we may use an information on $\rho_{\alpha}\left(\mathbf{R}_{i}\right)$ and $G_{\alpha \beta}\left(\mathbf{R}_{i}, \mathbf{R}_{j}\right)$ as an input for calculation of the surface properties. Since a flat wall problem is translationally invariant along the surface, we are interested in the contact values $\rho_{\alpha}\left(d_{\alpha} / 2\right)$ and $G_{\alpha \beta}\left(d_{\alpha \beta}\right)$, provided that the lattice spacing is not much different from the bead diameters.

The mean field approximation for the grand canonical potential (per lattice site) is

$$
\Omega=k T\left[\sum_{\alpha} \theta_{\alpha} \ln \theta_{\alpha}+\left[1-\sum_{\alpha} \theta_{\alpha}\right] \ln \left(1-\sum_{\alpha} \theta_{\alpha}\right)-\sum_{\alpha} \mu_{\alpha} \theta_{\alpha}-2 \sum_{\alpha, \beta} W_{\alpha \beta} \theta_{\alpha} \theta_{\beta}\right],
$$

where the partial coverage $\theta_{\alpha}$ is determined through the minimization of $\Omega$

$$
\frac{\partial \Omega}{\partial \theta_{\alpha}}=0
$$

This gives

$$
\mu_{\alpha}^{*}=\mu_{\alpha} / k T=\ln \theta_{\alpha}-\ln \left(1-\sum_{\alpha} \theta_{\alpha}\right)-4 \sum_{\beta} W_{\alpha \beta} \theta_{\beta}
$$


For the sake of simplicity we consider the case of vanishing lateral interaction $W_{\alpha \beta}=$ 0 . Although this is appropriate for dilute mixtures, we retain such an approximation in order to focus on an interplay between the interfacial segregation and the selective adsorption. Then the partial coverages are determined by the surface activities.

$$
\theta_{\alpha}=\frac{\mathrm{e}^{\mu_{\alpha}^{*}}}{1+\sum_{\alpha} \mathrm{e}^{\mu_{\alpha}^{*}}} .
$$

The selectivity coefficient $S_{B A}$, defined as a weighted difference of the coverages

$$
S_{B A}=c_{A} \theta_{B}-c_{B} \theta_{A}
$$

measures the surface excess of one component. The concentrations are introduced in order to ensure $S_{B A} \rightarrow 0$ as one of the components vanishes.

\section{Results and discussion}

Due to the singular shape of the adsorbing potential (1) the density profile consists of two parts: the regular and the singular one

$$
\rho_{\alpha}(z)=\rho_{\alpha}^{r}(z)+\rho_{\alpha}^{s}(z) .
$$

The singular part determines the number density of adsorbed particles, while the regular part describes a distribution of nonadsorbed species. The surface density excess $\Gamma_{\alpha}$ is given by

$$
\Gamma_{\alpha}=\int_{d_{\alpha} / 2}^{\infty}\left[\rho_{\alpha}(z)-\rho_{\alpha}^{\text {bulk }}\right] \mathrm{d} z
$$

Therefore, we deal with two ingredients coming from the splitting of the density profile (11). The integral of the singular part is associated with the surface coverage $\theta_{\alpha}$. The regular counterpart describes an accumulation of nonadsorbed particles, induced by the spatial confinement.

\subsection{Interfacial segregation without specific adsorption}

As is discussed above, all the relevant properties of the problem in question are expressed through the characteristics of the reference state, where there is no specific adsorption. A role of the spatial confinement is described by the surface density excess (an analogy of the regular part of $\Gamma_{\alpha}$ ). If the density oscillation falls off rapidly enough, then the arrangement of the species near the hard wall is determined by the wall-particle contact probabilities. The contact density is given by [8]:

$$
g_{\alpha}\left(d_{\alpha} / 2\right)=\varrho_{\alpha}\left(d_{\alpha} / 2\right) / \varrho_{\alpha}=\frac{1-\eta+3 d_{\alpha} \zeta_{2}}{[1-\eta]^{2}}-\frac{m_{\alpha}-1}{m_{\alpha}} \frac{1}{1-\eta},
$$

where $\zeta_{2}=\pi / 6 \sum_{\alpha} \varrho_{\alpha} d_{\alpha}^{2}$. The pair contact probability can be found in [11], but for the moment this quantity is irrelevant to our analysis. As is seen from equation (13), we deal with an interplay between the bead size $\left(d_{\alpha}\right)$ and the chain length 
$\left(m_{\alpha}\right)$, which determine a preferential accumulation of one species near the surface - the segregation. In order to estimate this effect the following expression for the segregation coefficient has been derived [8]:

$$
\Sigma_{B A}=\left(\varrho_{B}+\varrho_{A}\right)\left[\frac{3 \zeta_{2}}{(1-\eta)^{2}}\left(d_{B}-d_{A}\right)-\frac{1}{1-\eta}\left(\frac{m_{B}-m_{A}}{m_{B} m_{A}}\right)\right] .
$$

Note that the segregation coefficient is defined as $\left(\varrho_{B}+\varrho_{A}\right)\left(g_{B}\left(d_{B} / 2\right)-g_{A}\left(d_{A} / 2\right)\right)$, such that a role of the concentration difference is ignored, which is suitable for equimolar mixtures. Nevertheless, this does not change qualitatively our conclusions. As is seen $\Sigma_{B A}$ consists of two parts. The first of them is proportional to the difference of the bead diameters, while the second part gives a contribution coming from the difference in the chain lengths. The first contribution is dominant at high densities and decreases with decreasing density (i.e., when $\zeta_{2}$ and $\eta$ are small). This suggests that the segregation is mainly determined by the difference $d_{B}-d_{A}$ when the density is high enough. In this way the particles with larger bead diameter are segregated to the surface.

In contrast, the second term of equation (14) becomes significant at low densities. Based on this we conclude that the difference $m_{B}-m_{A}$ is a dominant segregation source at low densities. From the above equation it is also seen that $\Sigma_{B A}$ is similar to that of hard spheres if $m_{B}=m_{A}$ (not only when $m_{B}=m_{A}=1$ ). For the case of equal diameters $d_{B}=d_{A}$ the $\Sigma_{B A}$ is negative (if $m_{B}>m_{A}$ ). This reflects the depletion of longer chains near the wall, such that shorter chains are segregated to the surface.

It is interesting that the first term can be expressed through the diameter ratio $d=d_{B} / d_{A}$

$$
\zeta_{2}\left(d_{B}-d_{A}\right)=\frac{\eta\left(d+R d^{3}-R d^{2}\right)}{1+R d^{3}}, \quad R=c_{B} / c_{A}
$$

while the second one involves the chain lengths $m_{A}$ and $m_{B}$ separately. This agrees with the result found in the framework of the field theory [3].

Therefore, we deal with a competition of two effects. An increase of an aggregate size due to the segment diameter leads to the accumulation near the surface, while a growth of the chain length results in depletion.

\subsection{Selectivity due to specific adsorption}

Taking into account the adsorptive activity of the surface we may discuss several possible situations which can be analyzed through the behaviour of the selectivity coefficient

$$
S_{B A}=\frac{c_{A} \lambda_{B} \varrho_{B} g_{B}\left(d_{B} / 2\right) d_{B}^{3}-c_{B} \lambda_{A} \varrho_{A} g_{A}\left(d_{A} / 2\right) d_{A}^{3}}{1+\lambda_{B} \varrho_{B} g_{B}\left(d_{B} / 2\right) d_{B}^{3}+\lambda_{A} \varrho_{A} g_{A}\left(d_{A} / 2\right) d_{A}^{3}} .
$$

If the surface is indifferent to the species $\left(\lambda_{A}=\lambda_{B}=\lambda\right)$, then the selective adsorption is determined by the segregation process near the surface. In other words, the species that segregate to the surface are preferentially adsorbed. In this case 
the selectivity involves the interplay of the mixture composition and its geometrical parameters. For equimolar composition, $S_{B A}$ behaves essentially like $\Sigma_{B A}$. As is seen in figure 1, depending on a combination of these parameters, one may observe a preferential adsorption of one species at almost any composition, or $B$ replaces $A$ with increasing concentration. In order to analyze the chain length difference we set $m_{A}=m$ and $m_{B}=m+D$, such that $D$ gives the difference. If the segment sizes are equal $\left(d_{B}=d_{A}\right)$, then the shorter chains are preferentially adsorbed. This may seem to contradict the results [3] obtained within the field theory that predicts the opposite. First of all, it is worth noting that the depletion of longer chains results from the short range packing effects, while the field theory resolves the polymer structure on the scale of the gyration radius. The depletion effect is confirmed by the computer simulation data $[5,6]$ and the results used here have shown [8] a rather good agreement with those data. Secondly, here we ignore the pairwise interaction at the surface (recall, $\left.W_{\alpha \beta}=0\right)$. It is known [4], however, that the species with stronger lateral interaction are adsorbed preferentially. In this way we neglect the cooperative effects which result from the chain connectivity and should be pronounced when the coverage increases. This interaction is governed by the contact value of the radial distribution function $G_{\alpha \beta}\left(d_{\alpha \beta}\right)$ for the chain segments. In order to determine the role of this ingredient, let us consider the pair correlation in one component with the chain length $m$. Then, following [11], we have

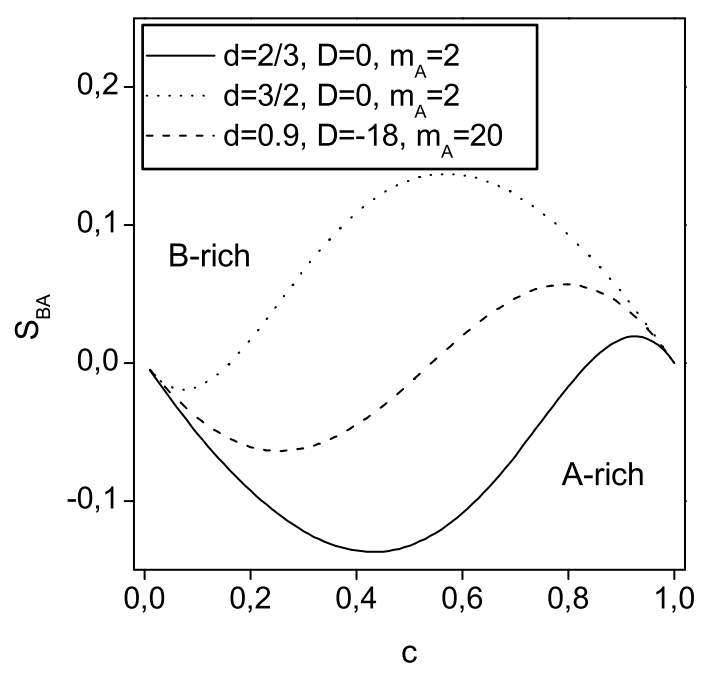

Figure 1. Selectivity coefficient $S_{B A}$ as function of $B$ concentration $\left(c=c_{B}\right)$ for $\eta=0.4$ and $\lambda_{A}=\lambda_{B}=1$.

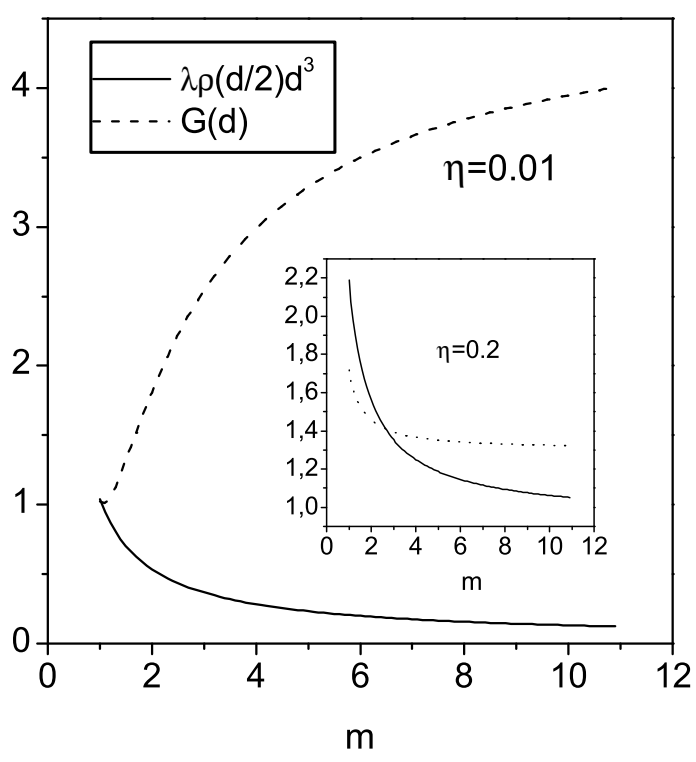

Figure 2. Dimensionless surface activity (solid) and the contact value of the radial distribution function (dashed) as functions of the chain length. The inset displays the high density behaviour.

$$
G(d)=\frac{1+\eta / 2}{(1-\eta)^{2}}-\frac{m-1}{m} \frac{1}{2(1-\eta)}+\frac{1}{24 \eta}\left[\frac{m-1}{m}\right]^{2} .
$$




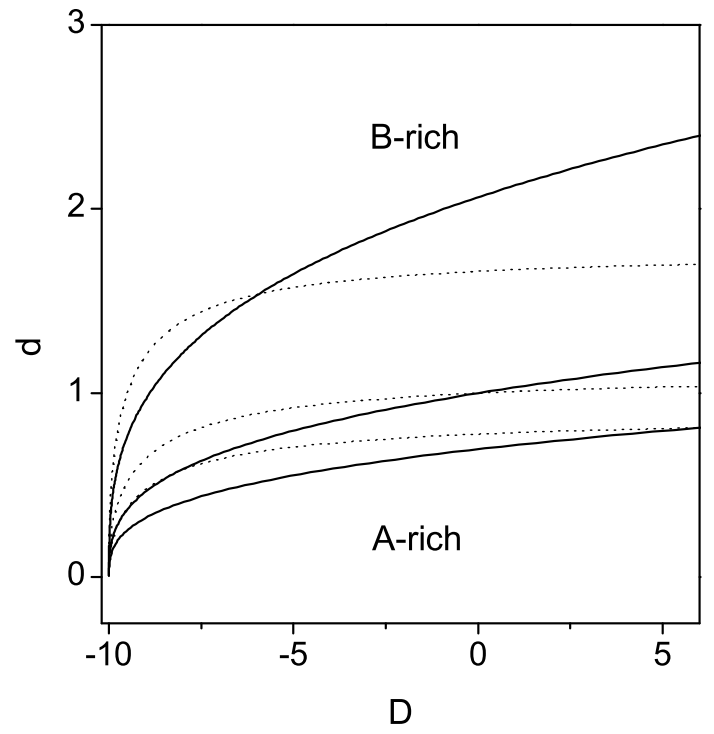

Figure 3. The $d-D$ diagram exhibiting the location of $A$ - and $B$-rich domains. Lines $(c=0.1,0.5,0.75$ from the top to the bottom) mark the boundary of zero selectivity. Other parameters are as follows: $\eta=0.1$ (solid), $\eta=0.4$ (dashed), $m_{A}=10, m_{B}=m_{A}+D, \lambda_{A}=\lambda_{B}=1$.

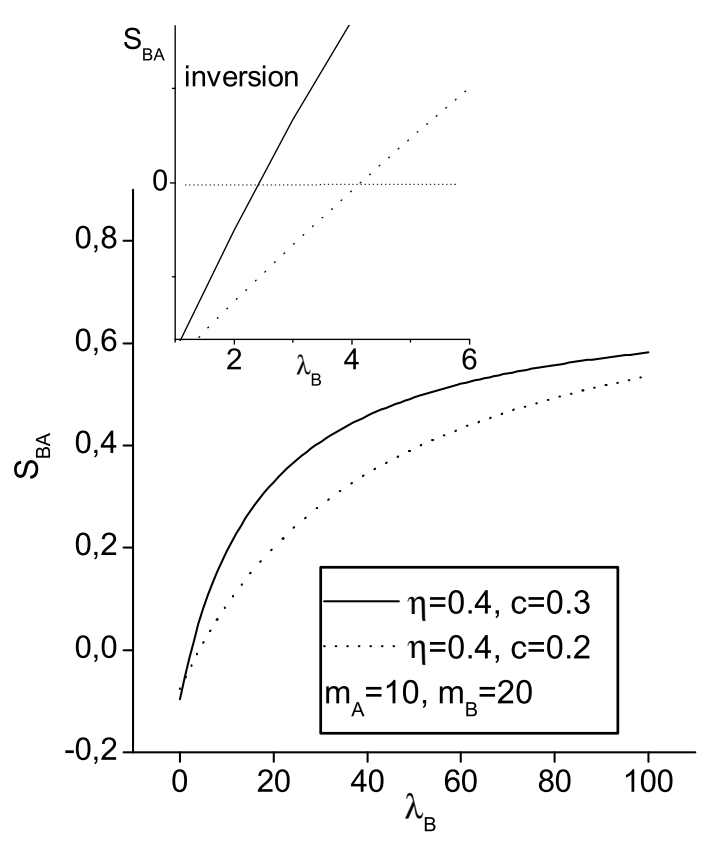

Figure 4. Selectivity coefficient as a function of $\lambda_{B}$ at fixed $\lambda_{A}=1$.
The first two terms correspond to the intermolecular correlation between the segments, while the third one represents the intramolecular correlation in a chain. Therefore, there are two driving forces for adsorption: the surface activity $\lambda \varrho g(d / 2)$ and the lateral interaction, related to $G(d)$. In figure 2 these quantities are plotted as functions of the chain length. At relatively high packing (see the inset) both quantities decrease with $m$. This suggests that the adsorption of the shorter chains should be promoted. At low densities the last term in equation (17) dominates and so $G(d)$, and consequently the lateral interaction, grows with $m$. In this domain the longer chain should replace the shorter ones. This implies the existence of some cross-over density at which the adsorption preference changes.

As is discussed above, the selectivity is driven by two competitive factors: the chain length difference and the segment diameter ratio. For a given set of the "collective" parameters (density and composition) there is a combination of the geometrical parameters $(d, m, D)$ at which the preferential adsorption disappears. Such a $d-D$ diagram is displayed in figure 3. The lines of zero selectivity separate the $B$ - and $A$-rich regions. Obviously, the $A$-rich region shrinks as the molar fraction of $B$ increases. The boundary between these two regions becomes less sensitive to the chain length difference with increasing packing fraction.

Now we analyze the selective adsorption when the surface is more active with respect to one component $\left(\lambda_{B} \neq\right.$ $\left.\lambda_{A}\right)$. In particular, we start from the conditions at which the $B$ component would be depleted in case of the indif- 
ferent surface and would increase $\lambda_{B}$ at fixed $\lambda_{A}=1$. The selectivity coefficient for this case is shown in figure 4 . It is seen that $S_{B A}$ changes the sign as $\lambda_{B}$ increases (see the inset). We deal with an interplay of two competing factors: the surface tends to adsorb $B$ which is depleted due to the interfacial segregation. Then the first process wins as some threshold activity is achieved. The threshold increases with decreasing concentration of $B$. The selectivity grows with the overall density $\eta$. The segregation layer with the depleted $B$ has a given thickness, while in the surface neighbourhood we have an inverted region (the inset), where $B$ dominates.

\section{Conclusion}

In this paper we have shown that the adsorption from polymer mixtures is associated with a variety of competing factors which favour the selectivity. If the surface is indifferent to the components, then the preferential adsorption is driven by the surface induced segregation of the mixture. The selectivity is mainly determined by the bead diameter ratio at high density, while the chain length difference is dominant at low densities. The surface selects the shorter chains with a larger bead diameter. The longer chains, however, can replace the shorter ones due to the increase of the lateral interaction with an increasing chain length. This effect is especially pronounced at low densities. If the surface is more active with respect to one of the components, then the depleted species can be preferentially adsorbed, forming the inverted region with the alternation of the $A$ - and $B$-rich fields across the interface. Such competing factors lead to the nonideality of polymer mixtures at the surface neighbourhood. In particular, one may expect the azeotropic effects [4], which are especially important in the course of the critical adsorption or the phase separation in the interfacial region.

The results obtained here may have several important applications, related to the separation of polymeric mixtures. In particular, some implications connected with the Chornobyl problem can be drawn. Some of the radioactive species (like uranyl or plutonyl) polymerize in the presence of water. These can be accumulated using properly designed adsorbents. Our results demonstrate that some optimal combination of the polymer size, mixture composition and the surface activity may significantly enhance this process.

\section{References}

1. Fleer G.J., Lyklema J. - In: Adsorption from solution at solid/liquid interface (G.D. Parfitt and C.H. Rochester Eds.). New York, Academic press, 1983.

2. Kawaguchi M., Takahashi A. // Adv. Colloidal and Interface Science, 1992, vol. 37, p. 219.

3. Baschnagel J., Johner A., Joanny J.-F. // Phys. Rev. E, 1997, vol. 55, p. 3072.

4. Heuchel M. // Langmuir, 1997, vol. 13, p. 1150.

5. Yethiraj A., Hall C.K. // J. Chem Phys., 1989, vol. 91, p. 4827.

6. Honnel K.G., Hall C.K. // J. Chem. Phys., 1991, vol. 95, p. 4481. 
7. Yethiraj A. // Phys. Rev. Lett., 1995, vol. 74, p. 2018.

8. Vakarin E.V. // J. Chem. Phys., 1998, vol. 109, p. 338.

9. Badiali J.P., Blum L., Rosinberg M.L. // Chem. Phys. Lett., 1986, vol. 129, p. 149.

10. Vakarin E.V., Holovko M.F., Duda Yu.Ya. // Mol. Phys., 1997, vol. 91, p. 203;

Vakarin E.V., Duda Yu.Ya., Holovko M.F. // J. Chem. Phys., 1997, vol. 107, p. 5569.

11. Chang J., Sandler S.I. // J. Chem. Phys., 1995, vol. 103, p. 3196.

\section{Селективна адсорбція з полімерної суміші на поверхні твердого тіла}

\section{Е.В.Вакарін, М.Ф.Головко}

Інститут фізики конденсованих систем НАН України, 79011 Львів, вул. Свєнціцького, 1

Отримано 17 жовтня 2001 р.

Селективна адсорбція з бінарної полімерної суміші на кристалічній поверхні вивчається в рамках моделі граткового газу в комбінації 3 результатами асоціативної теорії. Показано, що взаємовплив селективності та поверхнево-індукованої сегрегації приводить до різноманітних конкуруючих факторів, що визначають процес адсорбції. Проаналізовано роль діаметра сегмента, довжини ланцюга та поверхневої активності у переважній адсорбції одної компоненти. Обговорюється також зміна коефіцієнта селективності з густиною та концентраційним складом.

Ключові слова: адсорбція, селективність, полімерні суміші

PACS: $68.45 . D a$ 
